\title{
Dynamic Colonization of Klebsiella pneumoniae Isolates in Gastrointestinal Tract of Intensive Care Patients
}

\section{OPEN ACCESS}

Edited by:

Yi-Wei Tang,

Memorial Sloan Kettering Cancer

Center, United States

Reviewed by:

Michael Bachman,

University of Michigan, United States

Claudia Monari,

University of Perugia, Italy

${ }^{*}$ Correspondence:

Rong Zhang

zhang-rong@zju.edu.cr

Gong-Xiang Chen

chengongxiang@zju.edu.cn

Specialty section:

This article was submitted to Antimicrobials, Resistance

and Chemotherapy,

a section of the journal

Frontiers in Microbiology

Received: 17 October 2018

Accepted: 28 January 2019

Published: 11 February 2019

Citation:

Sun $Q$, Gu D, Wang Q, Hu Y, Shu L, Hu J, Zhang $R$ and Chen G-X

(2019) Dynamic Colonization of Klebsiella pneumoniae Isolates in Gastrointestinal Tract of Intensive Care Patients.

Front. Microbiol. 10:230. doi: 10.3389/fmicb.2019.00230

\section{Qiao-ling Sun ${ }^{1}$, Danxia $\mathrm{Gu}^{2}$, Qi Wang ${ }^{1}$, Yanyan $H u^{1}$, Lingbin Shu ${ }^{1}$, Jie $H u^{1}$, Rong Zhang ${ }^{1 *}$ and Gong-Xiang Chen ${ }^{1 *}$}

1 Department of Clinical Laboratory, Second Affiliated Hospital of Zhejiang University, School of Medicine, Hangzhou, China, ${ }^{2}$ Key Laboratory of Tumor Molecular Diagnosis and Individualized Medicine of Zhejiang Province, Clinical Research Institute, Zhejiang Provincial People's Hospital, People's Hospital of Hangzhou Medical College, Hangzhou, China

Gastrointestinal carriage is regarded as a major reservoir of $K$. pneumoniae infections, especially in intensive care patients. A total of 101 (95.3\%) KPC-producing carbapenemresistant $K$. pneumoniae (CRKP) isolates were identified among $106 \mathrm{CRKP}$ isolates collected from stool samples of inpatients performing active rectal screening for carbapenem-resistant Enterobacteriaceae during hospitalization in the ICUs of a tertiary hospital between 2016 and 2017. Among them, six KPC-producing CRKP isolates from three patients (two isolates for each patient) were identified with distinct antibacterial susceptibility. Our findings showed that: (1) bla $\mathrm{KPC}_{-2}$ gene is predominant in CRKP strains isolated from the intensive care patients and can be incorporated into various plasmids that are transmissible among multiple bacterial hosts in the human gastrointestinal tract; (2) the human gastrointestinal tract has a capacity to dynamically colonize multiple clones of CRKP strains with varied plasmids, diverse antimicrobial resistance genes and virulence genes. $K$. pneumoniae colonization is an important step in progression to extraintestinal infection, which provides the rationale for establishing intervention measures to prevent subsequent infection. Thus, close surveillance on CRKP colonization, together with effective infection prevention and control measures, should be put into practice.

Keywords: CRKP, KPC-2, gastrointestinal carriage, dynamical colonization, multiple clones

\section{INTRODUCTION}

Klebsiella pneumoniae is a major opportunistic pathogen that can cause invasive hospital-acquired infections among immune-compromised patients especially for those from ICU ward with critical illness. Carbapenem is a first line therapy for the treatment of infections caused by multidrugresistant K. pneumoniae. However, carbapenem-resistant $K$. pneumoniae (CRKP) has emerged as a public threat to cause serious infections with high mortality up to $33.24-50.06 \%$ (Xu et al., 2017). Recent data from the CHINET surveillance program showed that the prevalence of CRKP had dramatically increased from 3 to $17.9 \%$ since 2005 (Hu et al., 2016, 2017). A previous study on nationwide surveillance of clinical carbapenem-resistant Enterobacteriaceae (CRE) strains has 
revealed that $b l a_{\mathrm{KPC}-2}$ was the most prevalent genotype in China, accounting for $73 \%$ of CRKP isolates (Zhang et al., 2017).

Carbapenem-resistant Hypervirulent $K$. pneumoniae (CRHvKP), especially ST11 KPC-producing strains, have been increasingly reported in recent years (Zhang R. et al., 2015; Zhang Y. et al., 2015; Gu et al., 2018). They often cause invasive, even life-threatening infections with high mortality among young and healthy populations. In a previous study, we reported an outbreak of ST11 CR-HvKP in the ICU ward; further study indicated that the acquisition of a virulence plasmid carrying rmpA2 and aerobactin biosynthesis genes by classic ST11 CRKP strains played a critical role (Gu et al., 2018).

Gastrointestinal carriage has been regarded as a major reservoir of $K$. pneumoniae infections, especially in intensive care patients (Gorrie et al., 2017). A prospective study in 1971 indicated that $18.5 \%$ patients colonized with multidrug-resistant K. pneumoniae after hospital admission had higher risk to develop subsequent infection caused by identical bacteria within 21 days compared to those who did not become intestinal carriers (45\% vs. 11\%) (Martin and Bachman, 2018). A 2016 study reported similar colonization rates $(23 \%)$ and increased risk of infection following colonization (5.2\% in colonized vs. $1.3 \%$ in noncolonized) (Martin et al., 2016). The carriage rate of CRKP is reported to range from 8 to $9 \%$ in medical and surgical departments to $5 \%$ in intensive care units (WienerWell et al., 2010). CRKP colonization can persist and spread silently for years, even trigger a clonal outbreak in long-term care facilities while newly colonized patients can develop fatal infections (Martin and Bachman, 2018). Our previous study showed the evolution of tigecycline- and colistin-resistant CRKP has occurred in vivo under the antibiotic selection and CRKP could persistently colonize in the human gastrointestinal tract for 3 years even without antibiotic selection pressure (Zhang et al., 2018). Previous reports mainly focused on epidemiological data of CRKP carriage and associated risk factors (PapadimitriouOlivgeris et al., 2012; Feldman et al., 2013; PapadimitriouOlivgeris et al., 2013), in this study, we are going to investigate the molecular characterization of multiple clones of CRKP strains colonized in the gastrointestinal tract of intensive care inpatients, aiming at illustrating certain discipline underlying the colonization of CRKP.

\section{MATERIALS AND METHODS}

\section{The Rectal CRKP Isolates From the Intensive Care Inpatients}

A total of $106 \mathrm{~K}$. pneumoniae isolates that exhibited carbapenem resistance phenotype (MIC value of meropenem $\geq 4 \mu \mathrm{g} / \mathrm{ml}$ ) were identified from stool samples of inpatients performing active rectal screening for CRE during hospitalization in the ICUs of Second Affiliated Hospital of Zhejiang University, School of Medicine (Hangzhou, China) between 2016 and 2017. Identification of species was confirmed via a matrix-assisted laser desorption/ionization time-of-flight mass spectrometry (Bruker Daltonik GmbH, Bremen, Germany). The presence of carbapenem-resistant genes $\left(b l a_{\mathrm{NDM}}, b l a_{\mathrm{KPC}}, b l a_{\mathrm{IMP}}, b l a_{\mathrm{VIM}}\right.$, and $\left.b l a_{\mathrm{OXA}-48}\right)$ was screened using PCR (Zhang et al., 2017). Positive products were validated with Sanger DNA sequencing. A total of 101 (95.3\%) KPC-2-producing CRKP isolates were identified among 106 rectal CRKP isolates. Among them, six KPC-2-producing CRKP isolates from three patients (two isolates for each patient) were identified with distinct antibacterial susceptibility. In order to investigate the origin of the intestinal CRKP isolates and further look into the relationship between the rectal colonization and extraintestinal infections, we reviewed medical history of the three inpatients in detail and subjected the KPC-2-producing CRKP isolates collected during peri-hospitalization period to phenotypic and genotypic characterization.

\section{Phenotypic Characterization}

The antimicrobial susceptibilities of the isolates were determined using a broth microdilution procedure and the interpretations were in accordance with the guideline document M100-S26 established by Clinical and Laboratory Standards Institute (CLSI, 2016).

We did pulsed-field gel electrophoresis (PFGE), S1-PFGE, and Southern hybridization as previously reported (Huang et al., 2016). A dendrogram was generated from the homology matrix with a coefficient of $0.5 \%$ using the unweighted pair-group method using arithmetic averages ("UPGMA") to describe the relationships among PFGE profiles. Isolates were considered to belong to the same PFGE group if their Dice similarity index was $\geq 85 \%$.

The virulence gene $\operatorname{rmp} A 2$, encoded on the virulence plasmid, was identified by PCR as previously described (Gu et al., 2018). All isolates were performed with the string test to identify the hypermucoviscous phenotype as described previously ( $\mathrm{Gu}$ et al., 2018). As a test of virulence, we quantified virulence with Galleria mellonella larva models. The G. mellonella larva was injected into the hemocoel of each caterpillar via the last left proleg with $10 \mu \mathrm{l}$ suspensions of a $\mathrm{K}$. pneumoniae strain containing a final concentration of $10^{6} \mathrm{CFU} / \mathrm{mL}$, incubated at $37^{\circ} \mathrm{C}$ and observed every $12 \mathrm{~h}$ for 3 days. The effect of $1 \times 10^{6} \mathrm{CFU}$ of each $K$. pneumoniae strain on survival was assessed in G. mellonella. HvKP strain K. pneumoniae 4 and strain PC $K$. pneumoniae 4, reported in a previous study (Gu et al., 2018), were treated as controls. HvKP strain K. pneumoniae 4 was a ST11 KPC-producing hypermucoviscous strain harboring various virulence genes ( $r m p A 2$, iutA, iroE, $k p n, y c f M$, iucABCD, mrkABCDF, and fimA-H), located on the virulence plasmid pLVPK. Strain PC $K$. pneumoniae 4 is a mutant strain of $K$. pneumoniae 4 , of which the virulence plasmid has been removed in plasmid curing experiments. Strain PC K. pneumoniae 4 was negative for string test and demonstrated reduced virulence in G. mellonella models. Two control groups were performed: the first group was inoculated with PBS to monitor for killing due to physical trauma and attrition while the second received no injection. Eight larvae from each group were examined and all the experiments were performed in triplicates. Kaplan-Meier survival curves were plotted using GraphPad Prism version 7.00, the log rank (MantelCox) test was used to analyze whether significant differences 
$(P<0.05)$ in the survival rates of the infected $G$. mellonella larvae were observed.

\section{Whole Genome Sequencing and Bioinformatics Analysis}

Genomic DNA was extracted from overnight cultures by using the PureLink Genomic DNA Mini Kit (Invitrogen, Carlsbad, CA, United States) and was subjected to whole genome sequencing using $150 \mathrm{bp}$ pair-end strategies with the Illumina HiSeq X10 platform. Raw reads were trimmed and assembled to contigs using SPAdes v3.11.1 (Bankevich et al., 2012). Assembled genome sequences were submitted to the NCBI database with accession number QMKA00000000, QMKB00000000, QMKC00000000, QMKD00000000, QMKE00000000, and QMKF00000000. Genome sequences were annotated with the RAST tool (Overbeek et al., 2013) and Prokka (Seemann, 2014). Multilocus sequence types (MLSTs), virulence-associated genes encoding yersiniabactin, aerobactin, salmochelin and the regulators of mucoid phenotype were determined with Kleborate (Pichler et al., 2017). Serotyping was performed using Kaptive (Wyres et al., 2016). Acquired antibiotic resistance genes were identified with ResFinder 2.1 (Zankari et al., 2012). Heatmap of the antimicrobial resistance genes was generated using an in-house script.

\section{Ethics Statement}

The study was approved by the Ethics Committee of Second Affiliated Hospital of Zhejiang University, School of Medicine (2017-099). All subjects gave written informed consent in accordance with the Declaration of Helsinki.

\section{Biosafety Statement}

All concerns related to the safe and appropriate use of human-derived materials, infectious agents, or genetically modified organisms were approved by the Institutional Biosafety Committee of Second Affiliated Hospital of Zhejiang University, School of Medicine. All experiments were conducted under the guidelines from the Biological Agent Reference Sheet.

\section{RESULTS}

Three elderly patients aged 61-70 years were admitted to the ICU between August 2016 and February 2017. The three patients all developed diseases including bacterial pneumonia and bloodstream infections and underwent surgery, followed by antimicrobial treatment and mechanical ventilation, but they responded differently to antibiotic treatment. Only two patients, Patient 2 and 3, succeeded in infection control while Patient 1 experienced a persistent fever and pulmonary infection till against-advice discharge. Detailed information about the patients is available in the Figures 1, 2. Active rectal screening for CRE was conducted for all the patients during hospital admission and peri-hospitalization period, and all the three patients were found to carry the KPC-producing CRKP strains in the gastrointestinal tract on admission and during stays at healthcare settings. Thereinto, two CRKP isolates from stool samples of each patient presented distinct antimicrobial susceptibility profiles. In order to study the origin of the intestinal CRKP carriage and the relationship between the rectal colonization and extraintestinal infections, three, five, and five more KPC-producing CRKP isolates from various types of specimens including stool were successively identified in Patient 1 , Patient 2 , and Patient 3 during peri-hospitalization period, respectively (Figures 1, 2).

\section{Acquisition of KPC-Producing CRKP Isolates From Stool Samples}

Patient 1, a 71-year old woman, had previously been admitted to a local hospital for acute ventricular hemorrhage and was transferred to the Neurological intensive care unit (NICU) of our hospital on Aug 12, 2016. Three KPC-2-producing CRKP isolates (namely JZX2078, JZX2124, and JZX11-E8) were isolated. Isolate JZX2078 was recovered from stool sample after 1 week of admission, belonging to ST11 with unidentified serotype. It harbored three plasmids with sizes of $\sim 230, \sim 210$, and $\sim 80 \mathrm{~Kb}$. The $\sim 210 \mathrm{~Kb}$ plasmid was a pLVPK-like virulence plasmid which bears iucABCD, iroBCD, and rmpA2 genes. The $\sim 230 \mathrm{~Kb}$ plasmid was a pKP04VIM (KU318421.1)-like plasmid, while the $\sim 80 \mathrm{~Kb}$ one was a bla $a_{\mathrm{KPC}-2 \text {-bearing p69- }}$ 2 (CP025458.1)-like plasmid (Table 1). The second isolate JZX2124 was also isolated from stool a week before discharge. It was identified as a K19, ST1 strain which harbored two multidrug resistance plasmids, the $\sim 130 \mathrm{~Kb}$ p11219-CTXM (MF133442.1)-like plasmid and the $\sim 170 \mathrm{~Kb}$, bla $a_{\mathrm{KPC}-2}$-bearing pIT-06C07 (LT009688)-like plasmid (Table 1). Compared to isolate JZX2078 with 9 antibiotic resistance genes, isolate JZX2124 carried 12 resistance genes including tet $(A)$, oqx $A B$ genes and two different genes encoded for extended spectrum $\beta$-lactamases (ESBLs) as shown in Figure 3. And the third isolate JZX11-E8 was recovered from blood. With completely different antibacterial susceptibility profiles and distinct PFGE patterns (Figure 2), these three isolates were proved to originate from different clones.

Patient 2, a 66-year old man, was admitted to the Surgical Intensive Care Unit (SICU) for severe acute pancreatitis with acute respiratory distress syndrome on January 12th, 2017. Two weeks after admission, he was transferred to Surgical Ward preparing for a surgery of cholecystectomy and peritoneal lavage and drainage, immediately followed by transferring to the SICU and experiencing one more surgery of intraperitoneal hemostasis and peritoneal lavage and drainage. Two CRKP isolates, WJQ129 and WJQ196, were isolated from stool and sputum samples, respectively, during his staying in Surgical Ward, while three CRKP isolates (WJQ205, WJQ250, and WJQ298) were collected from stool, sputum, and drainage fluid samples, respectively, during his staying in SICU. With similar antimicrobial susceptibility profiles and PFGE patterns, isolates WJQ129 and WJQ196 shared high homology. The same situation was presented for isolates WJQ205, WJQ250, and WJQ298. However, as shown in Figure 2, isolates recovered from the patient during his earlier staying in Surgical Ward were completely distinct from those recovered during his later staying in SICU. It may be suggested that Patient 2 

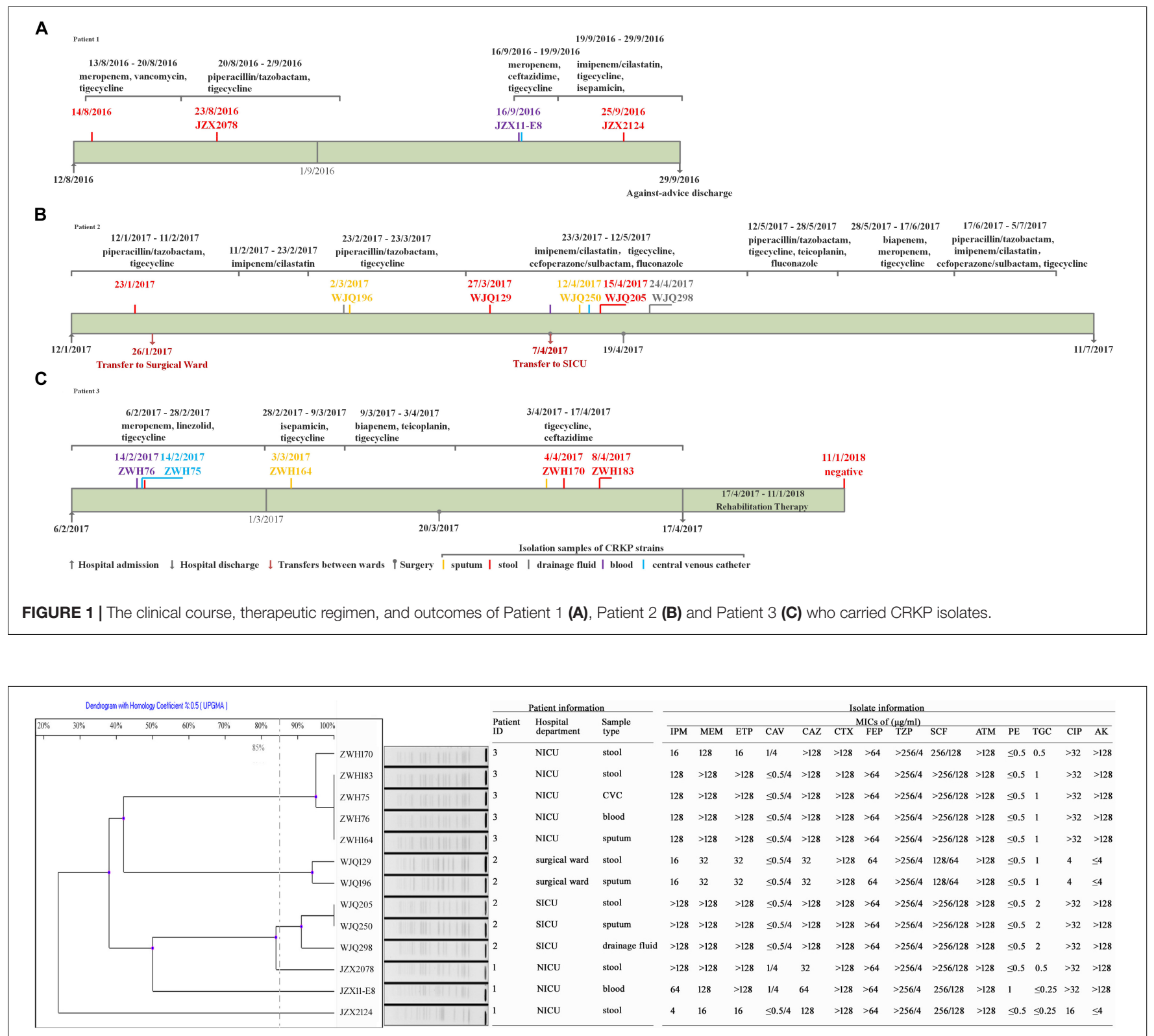

FIGURE 2 | Antimicrobial susceptibility and PFGE profiles of 13 CRKP isolates collected from three inpatients. IPM, Imipenem; MEM, Meropenem; ETP, Ertapenem; CAV, Ceftazidime-avibactam; CAZ, Ceftazidime; CTX, Cefotaxime; FEP, Cefepime; TZP, Piperacillin-tazobactam; SCF, Cefoperazone-sulbactam; ATM, Aztreonam; PE, colistin; TGC, Tigecycline; CIP, Ciprofloxacin; AK, Amikacin; CVC, central venous catheter.

TABLE 1 | Sequence information for rectal CRKP isolates from the three inpatients.

\begin{tabular}{|c|c|c|c|c|c|c|c|c|c|c|}
\hline Strain & Patient ID & $\begin{array}{l}\text { Number of } \\
\text { contigs } \\
>500 \text { bp }\end{array}$ & $\begin{array}{l}\text { Sequence } \\
\text { length (bp) }\end{array}$ & $\mathrm{G}+\mathrm{C}(\%)$ & N50 & MLST & Aerobactin & Salmochelin & Hypermucoidy & Serotype \\
\hline JZX2078 & 1 & 139 & 5863800 & 56.78 & 151743 & ST11 & $i U c A B C D$ & iroBCD & rmpA2 & unidentified \\
\hline JZX2124 & 1 & 425 & 6015115 & 56.7 & 202420 & ST1 & - & - & - & K19 \\
\hline WJQ129 & 2 & 149 & 5479176 & 57.13 & 217169 & ST290 & - & - & - & K21 \\
\hline WJQ205 & 2 & 231 & 5948640 & 56.71 & 99385 & ST11 & iucABCD & $\operatorname{iroBCD}$ & rmpA2 & K64 \\
\hline
\end{tabular}




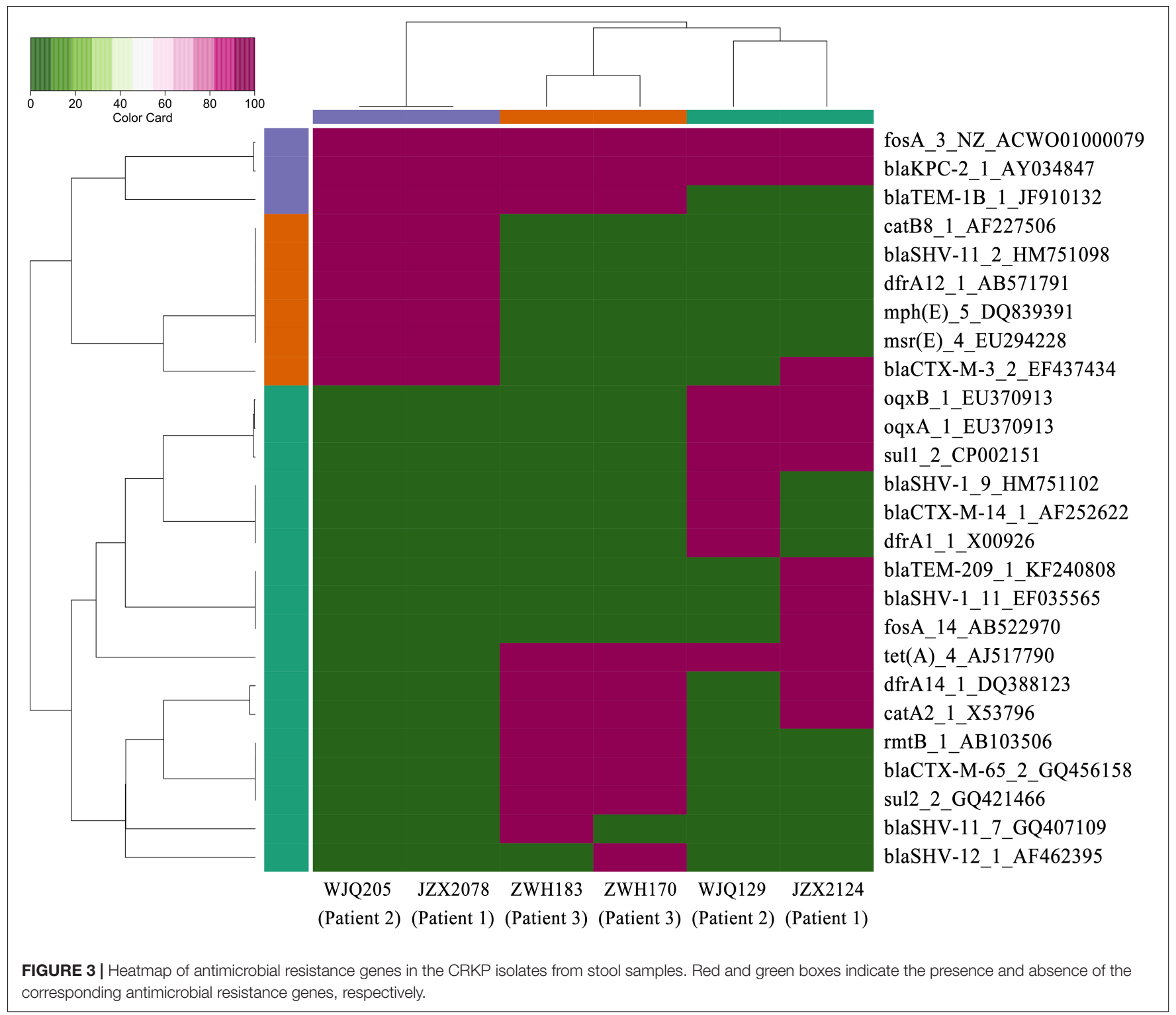

was successively infected by two different strains of KPCproducing $K$. pneumoniae isolated from different ICU wards. CRKP colonized in the gastrointestinal tract would easily cause extraintestinal infections once host defense system is weakened. Isolate WJQ129 was an ST290, K21 strain which harbored 9 antibiotic resistance genes (Table 1, Figure 3) and two plasmids with sizes of $\sim 230$ and $\sim 90 \mathrm{~Kb}$. The $\sim 230 \mathrm{~Kb}$ K. pneumoniae strain FDAARGOS_443 plasmid unnamed1 (CP023937.1)-like plasmid carries resistance genes tet(A), qnrS, $b l a_{C T X-M-14}$, and $d f r A 1$. The $\sim 90 \mathrm{~Kb}$ p628-KPC (KP987218.1)like plasmid carries bla $a_{\mathrm{KPC}-2}$. Isolate WJQ205 belonged to ST11, and identified as K64, carrying three plasmids ( $\sim 240$, $\sim 210$, and $\sim 80 \mathrm{~Kb}$ ) (Table 1). The $\sim 210 \mathrm{~Kb}$ plasmid is a pLVPK-like virulence plasmid, carrying iucABCD, iroBCD, and rmpA2 genes. The $\sim 240$ and $\sim 80 \mathrm{~Kb}$ plasmids are pA324-

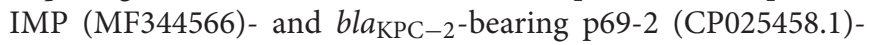
like plasmids, respectively.
Patient 3, a 61-year old woman, was admitted to our NICU for disorder of consciousness after a surgery of eliminating intracranial hematoma and bilateral external ventricular drainage in a local hospital. Five CRKP isolates were recovered from different samples belonging to two highly similar PFGE patterns. Interestingly, two homologous CRKP clones ZWH170 and ZWH183, collected from stool samples at short intervals, were both ST11, K64 serotype and harbored highly similar resistance genes, but showed different antibacterial susceptibility profiles (Table 1 and Figures 2, 3). Isolate ZWH170 carried plasmids with sizes of $\sim 138$ and $\sim 78 \mathrm{~Kb}$, while isolate ZWH183 carried one more plasmid which is around $\sim 105 \mathrm{~Kb}$. The $\sim 138 \mathrm{~Kb}$ plasmid was a pKPC-CR-HvKP4-like resistance plasmid carrying bla $a_{\mathrm{KPC}-2}$ and bla $\mathrm{CTX}-M-65$ genes. The $\sim 78 \mathrm{~Kb}$ plasmid was a p675920-2-like multidrug resistance plasmid carrying $b l a_{L A P-2}, q n r S 1$, tet(A), and sul2 genes. Additionally, the $\sim 105 \mathrm{~Kb}$ plasmid was a pLVPK-like virulence plasmid. 
After half-year rehabilitation therapy in another hospital, the patient agreed to provide the stool sample for follow-up investigation. Surprisingly, CRKP was no longer detected from her stool samples.

\section{Characterization of Virulence in CRKP Isolates}

Six KPC-producing K. pneumoniae isolates (JZX2078, JZX2124, WJQ129, WJQ205, ZWH170, and ZWH183) from stool samples of three patients were selected for further virulence characterization. According to the presence of virulence plasmid, the six isolates were divided into two groups: rmpA2-positive strains (JZX2078, WJQ205, and ZWH183) and rmpA2-negative strains (JZX2124, WJQ129, and ZWH170). S1-PFGE and Southern hybridization of the marker gene of the virulence plasmid $r m p A 2$, which was hybridized to the roughly $210 \mathrm{~Kb}$ and $105 \mathrm{~Kb}$ plasmids, confirmed the presence of the virulence plasmid in three of the ST11 KPC-producing CRKP isolates (JZX2078, WJQ205, and ZWH183). The 210 Kb pLVPK-like virulence plasmid, harboring $\operatorname{rmp} A 2$, iroBCD, and $i u c A B C D$ genes, were identified in two ST11 CRKP strains isolated from different ICU wards. The $\sim 105 \mathrm{~Kb}$ virulence plasmid in isolate
ZWH183, carrying the $\operatorname{rmp} A 2$, iucABCD genes was absent in isolate ZWH170, suggesting the attenuated virulence potential. The results of S1-PFGE and Southern hybridization were described in Figure 4.

All the six CRKP isolates from stool samples were negative for string test. Moreover, in G. mellonella larva models, the survival rate of larva infected with $r m p A 2$-carrying CRKP strains (JZX2078, WJQ205, and ZWH183) ranged from 20 to $40 \%$ at $16 \mathrm{~h}$ after infection; as for rmpA2-negative CRKP strains (JZX2124, WJQ129, and ZWH170), 60-80\% of the larva survived at $20 \mathrm{~h}$ (Figure 5). The control group infected with HvKP strain K. pneumoniae 4 resulted in $0 \%$ survival by $16 \mathrm{~h}$, whilst $70 \%$ survival was reached in larva infected with PC K. pneumoniae 4 after $16 \mathrm{~h}$. The survival rate of G. mellonella larvae infected with rmpA2-carrying CRKP strains (WJQ205 and ZHW183) was significantly lower than that infected with the counterpart rmpA2-negative CRKP strains (WJQ129 and ZWH170) $(P<0.05)$. However, there was no difference on the survival rate of $G$. mellonella larvae between the CRKP strains JZX2078 and JZX2124 $(P>0.05)$. The survival rate of $G$. mellonella larvae infected with rmpA2-carrying CRKP strains (WJQ205, ZHW183, and JZX2078) and rmpA2-negative CRKP strains (JZX2124, WJQ129, and ZWH170) showed

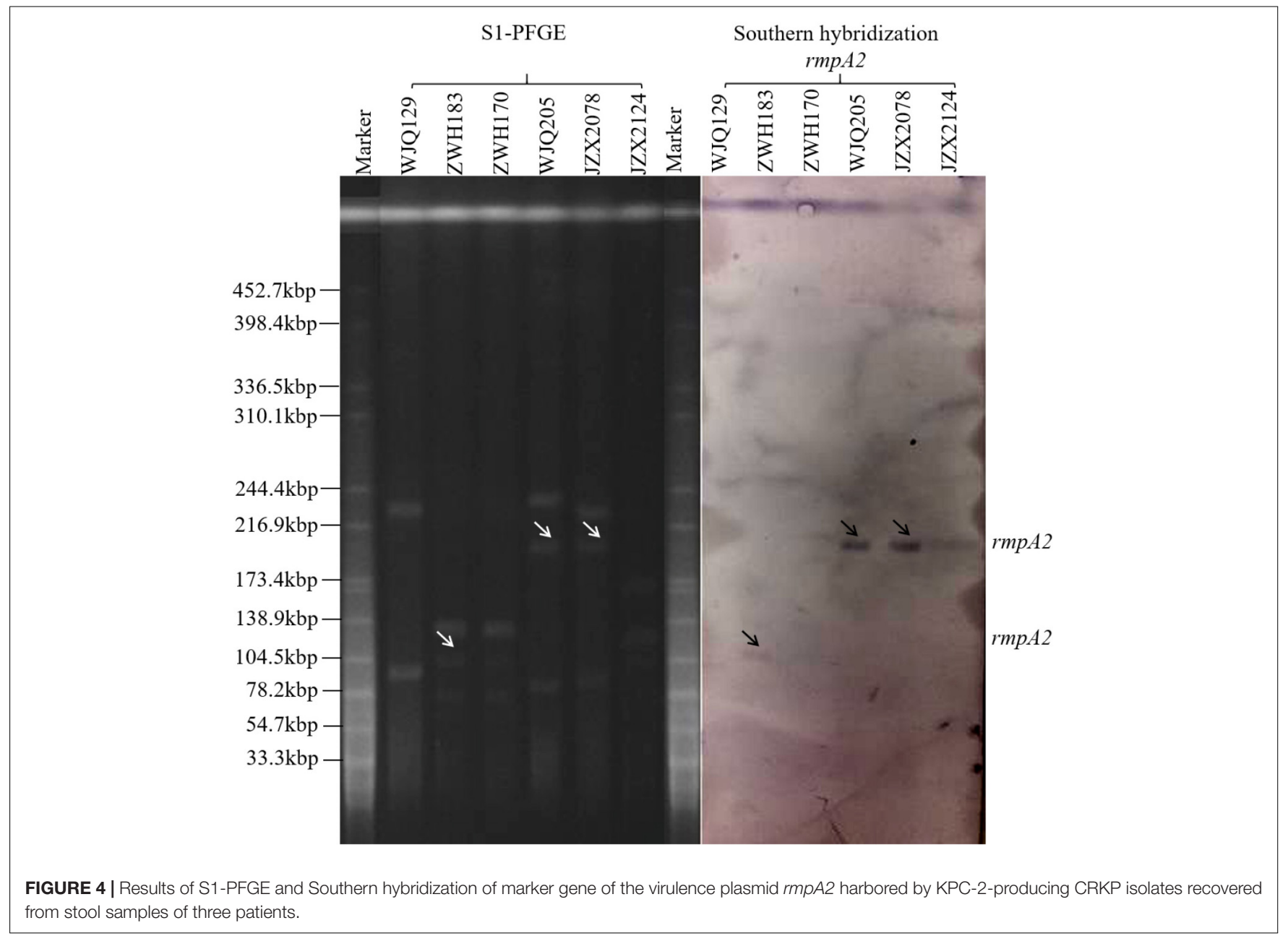


A

Patient 1

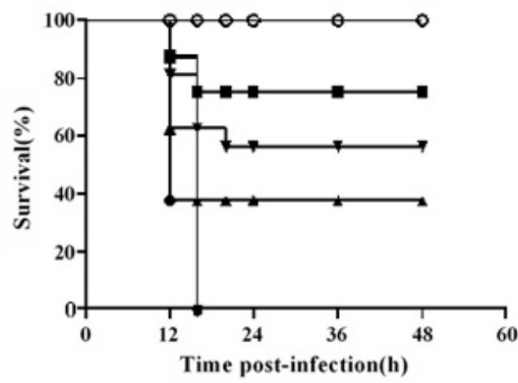

C

Patient 3
B

Patient 2

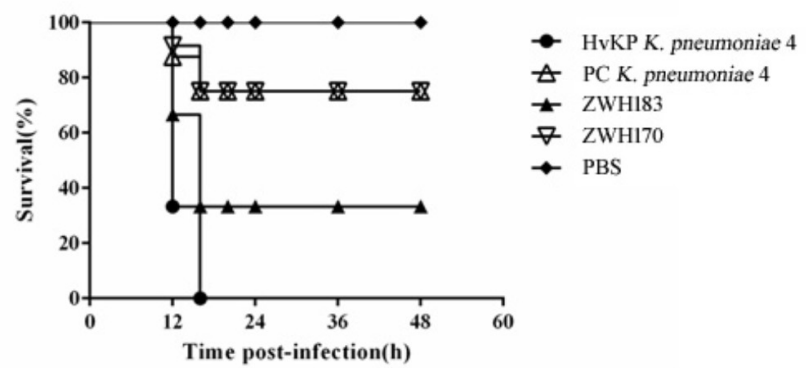

FIGURE 5 | Virulence potential of KPC-2-producing CRKP isolates from Patient 1 (A), Patient 2 (B) and Patient 3 (C) in the G. mellonella infection models. The effect of $1 \times 10^{6} \mathrm{CFU}$ of each K. pneumoniae strain on survival was assessed in G. mellonella. HvKP strain K. pneumoniae 4 was a ST11 KPC-producing hypermucoviscous strain harboring various virulence genes, located on the virulence plasmid pLVPK. Strain PC K. pneumoniae 4 is a mutant strain of K. pneumoniae 4, of which the virulence plasmid has been removed in plasmid curing experiments. Strain PC K. pneumoniae 4 was negative for string test and demonstrated reduced virulence in G. mellonella models.

statistical significance with that infected with $\operatorname{HvKP}(P<0.05)$. Interestingly, the survival rate of $G$. mellonella larvae infected with all the $r m p A 2$-carrying CRKP strains was significantly lower than that PC $K$. pneumoniae $4(P<0.05)$, while $r m p A 2$-negative CRKP strains all exhibited no difference on the survival rate of G. mellonella larvae with PC K. pneumoniae $4(P>0.05)$.

\section{DISCUSSION}

Gastrointestinal colonization is regarded as a common and significant reservoir of $K$. pneumoniae in terms of risk of transmission and infection (Martin and Bachman, 2018). Previous studies have found that K. pneumoniae gastrointestinal colonization was significantly associated with subsequent infections in inpatients (odds ratio $>4$ ), and $5 \%$ of colonized patients developed infections. In addition, $80 \%$ concordance was showed between infecting and colonizing isolates of K. pneumoniae within infected patients (Martin et al., 2016; Gorrie et al., 2017; Martin and Bachman, 2018). In our study, each of Patient 2 and Patient 3 had a homologous strain in the gut and an invasive site according to the Figure 2, suggesting that CRKP in the gastrointestinal tract would readily cause extraintestinal infections once the host defense system is weakened. Thus, CRKP colonization in the gastrointestinal tract is of significant importance.
As was widely reported (Holt et al., 2015; Lam et al., 2018), K. pneumoniae isolates are often volatile with a wide spectrum of diversity. The human gut is always considered as a reservoir for antibiotic resistance genes, with various species and abundant genes; horizontal transfer of resistance genes is extremely active. CRKP isolates colonized in the gut can be even more diversified. In the current study, three KPC-producing CRKP isolates from Patient 1 were highly heterogeneous belonging to different clones. Interestingly, the two from her intestine demonstrated completely different antibacterial susceptibility, PFGE and plasmid profiles. Patient 2 was successively infected by two different strains of CRKP isolated from different ICU wards, which indicated that hospitalacquired CRKP strains among the ICU ward is responsible for the colonizing and infecting strains in inpatients, and multiple clones of CRKP strains might spread in the clinical setting. bla $\mathrm{KPC}_{\mathrm{K}} 2$ gene was the predominant genotype in 95.3\% CRKP isolates and two bla $a_{\mathrm{KPC}-2 \text {-bearing plasmids }}$ belonged to two major types of $\sim 138 \mathrm{~Kb}$ and $\sim 80 \mathrm{~Kb}$ in size, suggesting that the $b l a_{\mathrm{KPC}-2}$ gene can be incorporated into various extrachromosomal elements which are capable of horizontal transfer among multiple bacterial hosts in the human gastrointestinal tract.

According to a previous report (Feldman et al., 2013), 74\% of the patients were identified with gastrointestinal carriage of CRKP 30 days after discharge from hospital, and when it came to 
6 months, the proportion declined to $<30 \%$. Persistent carriage of CRKP was associated with several risk factors, including catheter use, long-term care facilities, recent acquisition $(<4$ months), and a low functional status (Feldman et al., 2013). Additionally, a hypothesis was posed that CRKP could colonize in host cells to circumvent phagocytosis by immune cells, and survival of CRKP within host cells might serve as a reservoir to protect from antibiotic treatments and enable long-term coexistence with the host (Yang et al., 2018). In this study, all the three inpatients underwent mechanical ventilation, carried CRKP isolates in 2 weeks after hospital admission and had suffered from bloodstream infection caused by CRKP strains during hospitalization. The persistent carriage of CRKP in gastrointestinal tract could last for 1-6 months in our study, inferring that long-term hospital stay and prolonged antibiotic usage could acquire CRKP strains from the settings and enrich them in the host gastrointestinal tract, and CRKP might survive intracellularly to persist in the gastrointestinal colonization. Interestingly, after the half-year rehabilitation therapy in another hospital, CRKP was no longer detected from the stool samples of the Patient 3.

ST11 has been one of the most prevalent MLST in the clinical CRKP strains among different parts of the world. Previous study reported an outbreak of ST11 CR-HvKP in the ICU of our hospital, whose emergence was the result of acquisition of a $\sim 170 \mathrm{~Kb}$ virulence plasmid pLVPK carrying rmpA2, iroBCD, and iucABCD genes by classic ST11 CRKP strains (Gu et al., 2018). A 210 Kb pLVPK-like virulence plasmid carrying $r m p A 2$, iroBCD, and $i u c A B C D$ genes was identified in two ST11 KPC-producing CRKP isolates in the present study, which expressed lower virulence compared to that of the ST11 CR-HvKP previously described but higher virulence than that of the CRKP isolates without virulence plasmids. Notably, a comparison of the two homologous isolates $\mathrm{ZHW} 170$ and ZHW183 revealed that ZWH183 carried an additional virulence plasmid ( $\sim 105 \mathrm{~Kb}$, co-carrying the rmpA2 and iucABCD genes), which may be a glimpse of dynamic exchange of mobile elements in the gastrointestinal tract. Moreover, as was previously reported, CR-HvKP was found to account for only 3\% of infections caused by the ST11 CRKP strains across China (Gu et al., 2018). Consistently, most of CRKP isolates in this study are identified with low virulence. K. pneumoniae has a wider ecological distribution, significantly more varied DNA

\section{REFERENCES}

Bankevich, A., Nurk, S., Antipov, D., Gurevich, A. A., Dvorkin, M., Kulikov, A. S., et al. (2012). SPAdes: a new genome assembly algorithm and its applications to single-cell sequencing. J. Comput. Biol. 19, 455-477. doi: 10.1089/cmb.2012. 0021

CLSI (2016). Performance Standards for Antimicrobial Susceptibility Testing; Twenty-Sixth Informational Supplement. CLSI Document M100-S26. Wayne, PA: Clinical and Laboratory Standards Institute.

Feldman, N., Adler, A., Molshatzki, N., Navon-Venezia, S., Khabra, E., Cohen, D., et al. (2013). Gastrointestinal colonization by KPC-producing Klebsiella pneumoniae following hospital discharge: duration of carriage and risk factors for persistent carriage. Clin. Microbiol. Infect. 19, E190-E196. doi: 10.1111/ 1469-0691.12099 composition, greater antimicrobial resistance gene diversity and a higher plasmid load than other Gram negative opportunists, which means more opportunity to survive within and transfer between multiple environmental and animal-associated hosts; to capture plasmids from environmental microbial communities; to maintain antimicrobial resistance gene-carrying plasmids for long periods; and to transfer plasmids to other clinically important Gram negative bacteria (Wyres and Holt, 2018). Our finding showed that ST11 rmpA2-positive CRKP strains harbored three plasmids, of which two carried antimicrobial resistance genes and the third one was a virulence plasmid; rmpA2-negative CRKP strains only had two multidrug resistance encoding plasmids without virulence plasmid. It is suggested that the human gastrointestinal tract has a great capacity to colonize and enrich multiple clones of CRKP strains with varied plasmids as well as diverse antimicrobial resistance genes and virulence genes, which presumably due to frequent episodes of antibiotic treatment.

Colonization is believed as an important step in progression to extraintestinal infection which provides the rationale for establishing intervention measures to prevent subsequent infection by identifying the colonized patients. It is urgent matter to take the surveillance of rectal CRE carriage into routine test after admission.

\section{AUTHOR CONTRIBUTIONS}

QS did strain characterization and participated in manuscript writing. DG did the whole-genome sequencing and participated in manuscript writing. QW, YH, and JH participated in collecting the clinical data and strain characterization. LS did the Galleria mellonella infection experiments. RZ participated in the research design, data interpretation, and manuscript writing. G-XC designed and supervised the study, interpreted the data and wrote the manuscript.

\section{FUNDING}

This work was supported by the grants from National Natural Science Foundation of China (81601815, 81772250, and 81871705).

Gorrie, C. L., Mirceta, M., Wick, R. R., Edwards, D. J., Thomson, N. R., Strugnell, R. A., et al. (2017). Gastrointestinal carriage is a major reservoir of Klebsiella pneumoniae infection in intensive care patients. Clin. Infect. Dis. 65, 208-215. doi: $10.1093 / \mathrm{cid} / \mathrm{cix} 270$

Gu, D., Dong, N., Zheng, Z., Lin, D., Huang, M., Wang, L., et al. (2018). A fatal outbreak of ST11 carbapenem-resistant hypervirulent Klebsiella pneumoniae in a Chinese hospital: a molecular epidemiological study. Lancet Infect. Dis. 18, 37-46. doi: 10.1016/S1473-3099(17)30 489-9

Holt, K. E., Wertheim, H., Zadoks, R. N., Baker, S., Whitehouse, C. A., Dance, D., et al. (2015). Genomic analysis of diversity, population structure, virulence, and antimicrobial resistance in Klebsiella pneumoniae, an urgent threat to public health. Proc. Natl. Acad. Sci. U.S.A. 112, E3574-E3581. doi: 10.1073/pnas. 1501049112 
Hu, F. P., Guo, Y., Zhu, D. M., Wang, F., Jiang, X. F., Xu, Y. C., et al. (2016). Resistance trends among clinical isolates in China reported from CHINET surveillance of bacterial resistance, 2005-2014. Clin. Microbiol. Infect. 22(Suppl. 1), S9-S14. doi: 10.1016/j.cmi.2016.01.001

Hu, F. P., Guo, Y., Zhu, D. M., Wang, F., Jiang, X. F., Xu, Y. C., et al. (2017). CHINET surveillance of bacterial resistance across China: report of the results in 2016. Chin. J. Infect. Chemother. 17, 481-491.

Huang, Y., Yu, X., Xie, M., Wang, X., Liao, K., Xue, W., et al. (2016). Widespread dissemination of carbapenem-resistant Escherichia coli sequence type 167 strains harboring blaNDM-5 in clinical settings in China. Antimicrob. Agents Chemother. 60, 4364-4368. doi: 10.1128/AAC.00859-16

Lam, M. M. C., Wyres, K. L., Duchêne, S., Wick, R. R., Judd, L. M., Gan, Y. H., et al. (2018). Population genomics of hypervirulent Klebsiella pneumoniae clonal-group 23 reveals early emergence and rapid global dissemination. Nat. Commun. 9:2703. doi: 10.1038/s41467-018-05114-7

Martin, R. M., and Bachman, M. A. (2018). Colonization, infection, and the accessory genome of Klebsiella pneumoniae. Front. Cell. Infect. Microbiol. 8:4. doi: 10.3389/fcimb.2018.00004

Martin, R. M., Cao, J., Brisse, S., Passet, V., Wu, W., Zhao, L., et al. (2016). Molecular epidemiology of colonizing and infecting isolates of Klebsiella pneumoniae. mSphere 1:e00261-16. doi: 10.1128/msphere.00261-16

Overbeek, R., Olson, R., Pusch, G. D., Olsen, G. J., Davis, J. J., Disz, T., et al. (2013). The SEED and the Rapid Annotation of microbial genomes using Subsystems Technology (RAST). Nucleic Acids Res. 42, D206-D214. doi: 10. 1093/nar/gkt1226

Papadimitriou-Olivgeris, M., Marangos, M., Fligou, F., Christofidou, M., Bartzavali, C., Anastassiou, E. D., et al. (2012). Risk factors for KPC-producing Klebsiella pneumoniae enteric colonization upon ICU admission. J. Antimicrob. Chemother. 67, 2976-2981. doi: 10.1093/jac/dks316

Papadimitriou-Olivgeris, M., Marangos, M., Fligou, F., Christofidou, M., Sklavou, C., Vamvakopoulou, S., et al. (2013). KPC-producing Klebsiella pneumoniae enteric colonization acquired during intensive care unit stay: the significance of risk factors for its development and its impact on mortality. Diagn. Microbiol. Infect. Dis. 77, 169-173. doi: 10.1016/j.diagmicrobio.2013. 06.007

Pichler, C., Büchsel, M., Rossen, J. W., Vavra, M., Reuter, S., Kern, W. V., et al. (2017). First report of invasive liver abscess syndrome with endophthalmitis caused by a K2 serotype ST2398 hypervirulent Klebsiella pneumoniae in Germany, 2016. New Microbes New Infect. 17, 77-80. doi: 10.1016/j.nmni.2017. 02.006

Seemann, T. (2014). Prokka: rapid prokaryotic genome annotation. Bioinformatics 30, 2068-2069. doi: 10.1093/bioinformatics/btu153

Wiener-Well, Y., Rudensky, B., Yinnon, A. M., Kopuit, P., Schlesinger, Y., Broide, E., et al. (2010). Carriage rate of carbapenem-resistant Klebsiella pneumoniae in hospitalised patients during a national outbreak. J. Hosp. Infect. 74, 344-349. doi: 10.1016/j.jhin.2009.07.022

Wyres, K. L., and Holt, K. E. (2018). Klebsiella pneumoniae as a key trafficker of drug resistance genes from environmental to clinically important bacteria. Curr. Opin. Microbiol. 45, 131-139. doi: 10.1016/j.mib.2018.04.004

Wyres, K. L., Wick, R. R., Gorrie, C., Jenney, A., Follador, R., Thomson, N. R., et al. (2016). Identification of Klebsiella capsule synthesis loci from whole genome data. Microb. Genom. 2:e000102. doi: 10.1099/mgen.0.000102

Xu, L., Sun, X., and Ma, X. (2017). Systematic review and meta-analysis of mortality of patients infected with carbapenem-resistant Klebsiella pneumoniae. Ann. Clin. Microbiol. Antimicrob. 16:18. doi: 10.1186/s12941-017-0191-3

Yang, Z. Q., Huang, Y. L., Zhou, H. W., Zhang, R., and Zhu, K. (2018). Persistent carbapenem-resistant Klebsiella pneumoniae: a Trojan horse. Lancet Infect. Dis. 18, 22-23. doi: 10.1016/S1473-3099(17)30627-8

Zankari, E., Hasman, H., Cosentino, S., Vestergaard, M., Rasmussen, S., Lund, O., et al. (2012). Identification of acquired antimicrobial resistance genes. J. Antimicrob. Chemother. 67, 2640-2644. doi: 10.1093/jac/ dks261

Zhang, R., Dong, N., Huang, Y., Zhou, H., Xie, M., Chan, E. W., et al. (2018). Evolution of tigecycline- and colistin-resistant CRKP (carbapenem-resistant Klebsiella pneumoniae) in vivo and its persistence in the GI tract. Emerg. Microbes Infect. 7:127. doi: 10.1038/s41426-018-0129-7

Zhang, R., Lin, D., Chan, E. W., Gu, D., Chen, G. X., and Chen, S. (2015). Emergence of carbapenem-resistant serotype $\mathrm{K} 1$ hypervirulent Klebsiella pneumoniae strains in China. Antimicrob. Agents Chemother. 60, 709-711. doi: 10.1128/AAC.02173-15

Zhang, Y., Zeng, J., Liu, W., Zhao, F., Hu, Z., Zhao, C., et al. (2015). Emergence of a hypervirulent carbapenem-resistant Klebsiella pneumoniae isolate from clinical infections in China. J. Infect. 71, 553-560. doi: 10.1016/j.jinf.2015.07.010

Zhang, R., Liu, L., Zhou, H., Chan, E. W., Li, J., Fang, Y., et al. (2017). Nationwide surveillance of clinical carbapenem-resistant Enterobacteriaceae (CRE) strains in China. EBioMedicine 19, 98-106. doi: 10.1016/j.ebiom.2017.04.032

Conflict of Interest Statement: The authors declare that the research was conducted in the absence of any commercial or financial relationships that could be construed as a potential conflict of interest.

Copyright (c) 2019 Sun, Gu, Wang, Hu, Shu, Hu, Zhang and Chen. This is an open-access article distributed under the terms of the Creative Commons Attribution License (CC BY). The use, distribution or reproduction in other forums is permitted, provided the original author(s) and the copyright owner(s) are credited and that the original publication in this journal is cited, in accordance with accepted academic practice. No use, distribution or reproduction is permitted which does not comply with these terms. 